\title{
Bivariate Polynomials Modulo Composites and Their Applications
}

\author{
Dan Boneh and Henry Corrigan-Gibbs \\ Stanford University, Stanford CA 94305, USA
}

\begin{abstract}
We investigate the hardness of finding solutions to bivariate polynomial congruences modulo RSA composites. We establish necessary conditions for a bivariate polynomial to be one-way, second preimage resistant, and collision resistant based on arithmetic properties of the polynomial. From these conditions we deduce a new computational assumption that implies an efficient algebraic collision-resistant hash function. We explore the assumption and relate it to known computational problems. The assumption leads to (i) a new statistically hiding commitment scheme that composes well with Pedersen commitments, (ii) a conceptually simple cryptographic accumulator, and (iii) an efficient chameleon hash function.
\end{abstract}

Keywords: Algebraic curves, bivariate polynomials, cryptographic commitments, Merkle trees.

\section{Introduction}

In this paper, we investigate the cryptographic properties of bivariate polynomials modulo random RSA composites $N=p q$. We ask: for which integer polynomials $f \in \mathbb{Z}[x, y]$ does the function $f: \mathbb{Z}_{N} \times \mathbb{Z}_{N} \rightarrow \mathbb{Z}_{N}$ defined by $f$ appear to be a one-way function, a second-preimage-resistant function, or a collisionresistant function? We say that a polynomial $f \in \mathbb{Z}[x, y]$ is one-way if the function $f: \mathbb{Z}_{N} \times \mathbb{Z}_{N} \rightarrow \mathbb{Z}_{N}$ defined by $f$ is one-way (Section 3.1). We similarly define second-preimage-resistance (Section 3.2) and collision-resistance (Section 3.3) of polynomials $f \in \mathbb{Z}[x, y]$.

Using tools from algebraic geometry we develop a heuristic for deducing the cryptographic properties of a bivariate polynomial over $\mathbb{Z}_{N}$ from its arithmetic properties, namely from its properties as a polynomial over the rationals $\mathbb{Q}$. We give a number of necessary conditions for a bivariate polynomial to be oneway, second-preimage-resistant, or collision-resistant. We also provide examples of polynomials $f$ that appear to satisfy each of these properties and we offer separations between these three classes.

Taking collision resistance as an example, we conjecture that a bivariate polynomial $f \in \mathbb{Z}[x, y]$ that defines an injective function $f: \mathbb{Q}^{2} \rightarrow \mathbb{Q}$ gives a collision resistant function $f: \mathbb{Z}_{N}^{2} \rightarrow \mathbb{Z}_{N}$ where $N$ is a random RSA modulus of secret factorization (see Section 3.3). Constructing an explicit polynomial 
$f \in \mathbb{Z}[x, y]$ that is provably injective over the rationals is an open number theoretic problem [30. However, even relatively simple polynomials appear to be injective over $\mathbb{Q}^{2}$. For example, Don Zagier [13/34 conjectures that the polynomial $f_{\mathrm{zAG}}(x, y):=x^{7}+3 y^{7}$, which we refer to as the Zagier polynomial, is injective over the rationals. Since the only apparent efficient strategy for finding collisions in $f_{\text {zAG }}$ over $\mathbb{Z}_{N}$ is to find rational collisions and reduce them modulo $N$, we conjecture that $f_{\text {zAG }}$ is collision resistant over $\mathbb{Z}_{N}$. To build confidence in the assumption that $f_{\text {zAG }}$ is collision resistant over $\mathbb{Z}_{N}$ we discuss potential collisionfinding strategies and relate them to existing number theoretic problems.

Applications. We demonstrate that the existence of low-degree collision-resistant bivariate polynomials gives rise to very efficient instantiations of a number of cryptographic primitives.

First, we derive a statistically hiding commitment scheme which is computationally inexpensive to evaluate and composes naturally with Pedersen commitments. By "nesting" these new commitments inside of Pedersen commitments, we obtain an efficient zero-knowledge protocol for proving knowledge of an opening of a commitment which is nested inside of another commitment. Use of nested commitments reduces the length of transactions in an anonymous e-cash scheme 24] by roughly $70 \%$.

Second, we demonstrate that the new commitment scheme, in conjunction with Merkle trees, can serve as a simple replacement for one-way accumulators. Though the communication complexity of our accumulator construction is asymptotically worse than that of strong-RSA accumulators 8$]-O(\log |S|)$ versus $O(1)$ for a set $S$ being accumulated - our construction has the benefit of being conceptually simple and easy to implement.

Third, from the same collision-resistant polynomial, we derive a new chameleon hash function, signature scheme, claw-free permutation family, and a variablelength algebraic hash function.

\section{Related Work}

Multivariate polynomials in $\mathbb{Z}_{N}$ have a long history in cryptography. For example, the security of the Ong-Schnorr-Shamir signature scheme [26] followed from the hardness of finding solutions to a particular type of bivariate polynomial equation over $\mathbb{Z}_{N}$. Pollard and Schnorr later demonstrated a general attack against the hardness of finding solutions to such equations [28].

Shamir related the hardness of factoring certain multivariate polynomials modulo $N$ to the problem of factoring the modulus $N$ itself 33 . Schwenk and Eisfeld proposed encryption and signature schemes reliant on the hardness of finding roots of random univariate polynomials $f \in \mathbb{Z}[x]$ modulo a composite $N$, and they prove that this problem is as hard as factoring $N$ [31].

This work introduces a new statistically hiding commitment scheme based on low-degree polynomials. Commitment schemes are used widely in cryptography. 
Prior work has derived statistically hiding commitment schemes from the discrete log problem [27, the Paillier cryptosystem [12], and the RSA problem [3]. Verifying the correctness of opening a commitment in these existing schemes requires expensive modular exponentiations or elliptic curve scalar multiplications. Verifying an opening with our new commitment scheme requires just a few modular multiplications. By combining our new commitment scheme with traditional Pedersen commitments, we improve the communication efficiency of the Zerocoin decentralized e-cash construction [24].

Given a Pedersen commitment and a finite set of elements $S$, our commitment scheme leads to a simple zero-knowledge protocol for proving knowledge of an opening $x$ of the commitment such that $x \in S$. The length of the proof is $\log |S|$. This technique, which uses Merkle trees 21, has applications to anonymous authentication and credential systems and it has the potential to replace traditional RSA one-way accumulators, introduced by Benaloh and De Mare [5] and revisited by Barić and Pfitzmann [4].

Camenisch and Lysyanskaya presented an efficient zero-knowledge protocol which proves that a value contained in a Pedersen commitment is also contained in a particular strong-RSA accumulator [8]. The Camenisch-Lysyanskaya accumulator produces a shorter proof of knowledge than ours does, but the conceptual simplicity and ease of implementation may make our Merkle-style proof more attractive for some applications.

The "zero-knowledge sets" of Micali, Rabin, and Kilian solve an orthogonal problem: a prover publishes a commitment to a set $S$ and later can prove that $x \in$ $S$ without leaking other information about $S[23$. In contrast, we are interested in hiding the value $x$ but allow the set of items $S$ to be public.

\section{Cryptographic Properties of Polynomials}

We begin by surveying the cryptographic properties of integer polynomials modulo random RSA composites. Our goal is to relate the algebraic properties of polynomials to their cryptographic complexity. In particular, we identify families of integer polynomials that give rise to progressively stronger cryptographic primitives: one-way functions, second-preimage-resistant functions, and collisionresistant functions.

Notation. We write $x \stackrel{R}{\leftarrow} S$ to indicate that the variable $x$ takes on a value sampled independently and uniformly at random from a finite set $S$. A function $f: \mathbb{Z} \rightarrow \mathbb{R}^{+}$is negligible if it is smaller than $1 / p(\lambda)$ for every polynomial $p()$ and all sufficiently large $\lambda$. We denote an arbitrary negligible function in $\lambda$ as $\operatorname{neg}(\lambda)$. We use the notation $f(x):=x^{2}$ to indicate the definition of a new term.

In what follows, we let $\operatorname{RSAgen}(\lambda)$ denote a randomized algorithm that runs in time polynomial in $\lambda$. The algorithm generates two random len $(\lambda)$-bit primes $p$ and $q$ and outputs $(p, q, N:=p \cdot q)$. Here len $: \mathbb{Z}^{+} \rightarrow \mathbb{Z}^{+}$is some fixed function that determines the size of the primes $p$ and $q$ as a function of $\lambda$. 
Let $f \in \mathbb{Z}[x, y]$ be a bivariate polynomial. For $c \in \mathbb{Z}$ consider the curve $f(x, y)=c$. The genus of this curve is a standard measure of its "complexity:" conics have genus zero, elliptic curves have genus one, and so on (see, e.g. [218]). We define the genus of a polynomial $f$ as follows:

Definition 1. The genus of a polynomial $f \in \mathbb{Z}[x, y]$ is defined as

$$
\max _{c \in \mathbb{Q}}(\operatorname{genus}(f(x, y)=c)) \text {. }
$$

As we will see, the genus of a polynomial $f$ has some relation to its cryptographic properties. While we focus on bivariate polynomials, most of the following discussion generalizes to multivariates.

We use the following terms throughout this section to describe relationships between curves. (For more precise definitions, see Hindry and Silverman [18, Sec. A.1.2].) A rational map from a curve $C$ to another curve $C^{\prime}$ is a pair of rational functions $g$ and $h$ mapping points $(x, y)$ on $C$ to points $(g(x, y), h(x, y))$ on $C^{\prime}$. A birational map from $C$ to $C^{\prime}$ is a rational map which is a bijection between points on $C$ and $C^{\prime}$ such that the map's inverse is also rational. Two curves $C$ and $C^{\prime}$ and are birationally equivalent if there is a birational map from $C$ to $C^{\prime}$. An automorphism is a birational map from a curve to itself.

\subsection{One-Way Polynomials}

One-way functions are the basis of much of cryptography. A function $g: X \rightarrow Y$ is one-way if, given the image $c=f(x)$ of a random point $x \in X$, it is hard to find an $x^{\prime}$ such that $f\left(x^{\prime}\right)=c$. We first ask: what polynomials give rise to one-way functions?

Definition 2. A polynomial $f$ in $\mathbb{Z}\left[x_{1}, \ldots, x_{\ell}\right]$ is one-way if for every p.p.t. algorithm $\mathcal{A}$ the following advantage is a negligible function of $\lambda$ :

$$
\begin{aligned}
\operatorname{Adv}_{\mathcal{A}, f}(\lambda):=\operatorname{Pr}\left[N \leftarrow \operatorname{RSAgen}(\lambda), \bar{x} \stackrel{R}{\leftarrow}\left(\mathbb{Z}_{N}\right)^{\ell}, c \leftarrow f(\bar{x}):\right. & \\
& \left.\quad f(\mathcal{A}(N, c))=c \text { in } \mathbb{Z}_{N}\right] .
\end{aligned}
$$

Clearly linear polynomials are not one-way. A result of Pollard and Schnorr [28] shows that quadratic polynomials, indeed all genus zero polynomials, are not one-way.

Theorem 3. A genus zero polynomial $f \in \mathbb{Z}[x, y]$ is not one-way.

Proof sketch. For all $c \in \mathbb{Q}$ the curve $f(x, y)=c$ is of genus zero, or is a product of genus zero curves. A genus zero curve is birationally equivalent to a linear or quadratic curve $\tilde{f}(x, y)=0$ [18, Theorem A.4.3.1]. If $\tilde{f}(x, y)$ is linear in one of the variables $x$ or $y$ then finding points on this curve is easy thereby breaking the one-wayness of $f$. This leaves the case where $\tilde{f}(x, y)$ is quadratic in both $x$ and $y$. Let $N$ be an output of $\operatorname{RSAgen}(\lambda)$. Let $\tilde{f} \in \mathbb{Z}[x, y]$ be a quadratic polynomial in $x$ and $y$ and let $c \in \mathbb{Z}_{N}$. There is an efficient algorithm that for most $c \in \mathbb{Z}_{N}$ finds an $\left(x_{0}, y_{0}\right) \in \mathbb{Z}_{N}^{2}$ such that $\tilde{f}\left(x_{0}, y_{0}\right)=c$ in $\mathbb{Z}_{N}$, breaking the one-wayness of $f$. See for example [6, Sec. 5.2] for a description of the algorithm. 
Theorem 3 played an important role in analyzing the security of the OngSchnorr-Shamir signature scheme [26]. The scheme depended on the difficulty of finding solutions $(x, y)$ to the equation:

$$
x^{2}+a y^{2}=b \quad \text { in } \mathbb{Z}_{N}
$$

for known constants $a, b \in \mathbb{Z}_{N}$. Since this equation defines a genus-zero curve, Theorem 3 shows that it is possible to efficiently find solutions without knowledge of the factors of $N$. Pollard and Schnorr demonstrated an attack against the scheme soon after its publication [2832].

One-way Polynomials. It is not known how to break the one-wayness of polynomials $f \in \mathbb{Z}[x, y]$ that are not genus zero. Thus, for example, even a simple polynomial such as $f(x, y)=y^{2}-x^{3}$ may be one-way, although that would require further study.

\subsection{Second Preimage Resistant Polynomials}

A function $f: U \rightarrow V$ is second preimage resistant if, given $u \in U$, it is difficult to find a $u^{\prime} \neq u \in U$ such that $f(u)=f\left(u^{\prime}\right)$. We define a similar notion for polynomials:

Definition 4. A polynomial $f$ in $\mathbb{Z}\left[x_{1}, \ldots, x_{\ell}\right]$ is second preimage resistant if, for every p.p.t. algorithm $\mathcal{A}$, the following advantage is a negligible function of $\lambda$ :

$$
\begin{aligned}
\operatorname{Adv}_{\mathcal{A}, f}(\lambda):=\operatorname{Pr}\left[N \leftarrow \operatorname{RSAgen}(\lambda), \bar{x} \stackrel{R}{\leftarrow}\left(\mathbb{Z}_{N}\right)^{\ell}, \bar{x}^{\prime} \leftarrow \mathcal{A}(N, \bar{x}):\right. & \\
& \left.f(\bar{x})=f\left(\bar{x}^{\prime}\right) \text { in } \mathbb{Z}_{N} \wedge \bar{x} \neq \bar{x}^{\prime}\right] .
\end{aligned}
$$

Since genus 0 polynomials are not one-way they are also not second preimage resistant. It is similarly straight-forward to show that no genus-one polynomial is second preimage resistant.

Proposition 5. A genus one polynomial $f \in \mathbb{Z}[x, y]$ is not second preimage resistant.

To see why, let $f \in \mathbb{Z}[x, y]$ be a polynomial such that $f(x, y)=c$ is a curve of genus one for all but finitely many $c \in \mathbb{Q}$. Then $f$ is not second preimage resistant because of the group structure on elliptic curves. That is, let $N$ be an output of RSAgen $(\lambda)$. Choose a random pair $\left(x_{0}, y_{0}\right) \in \mathbb{Z}_{N}^{2}$ and set $c:=f\left(x_{0}, y_{0}\right) \in \mathbb{Z}_{N}$. Then $P=\left(x_{0}, y_{0}\right)$ is a point on the curve $f(x, y)=c$ and so is the point $2 P=P+P$ where addition refers to the elliptic curve group operation. With overwhelming probability $2 P$ is not the point at infinity and therefore, given $P$ as input, the adversary can output $2 P$ as a second preimage for $P$. It follows that $f$ is not second preimage resistant.

Even polynomials that give higher genus curves need not be second preimage resistant. For example, a hyperelliptic polynomial of genus $g \geq 2$ has the form 
$f(x, y)=y^{2}-h(x) \in \mathbb{Z}[x, y]$ where $h \in \mathbb{Z}[x]$ is a polynomial of degree $2 g+1$ or $2 g+2$. The simple fact that $f\left(x_{0}, y_{0}\right)=f\left(x_{0},-y_{0}\right)$ immediately gives a second preimage attack on these polynomials: given $\left(x_{0}, y_{0}\right)$ the attacker outputs $\left(x_{0},-y_{0}\right)$ as a second preimage.

The fact that all curves of genus two are hyperelliptic [18, Theorem A.4.5.1] leads to the following proposition:

Proposition 6. A genus two polynomial $f \in \mathbb{Z}[x, y]$ is not second preimage resistant.

This proposition, in combination with Theorem 3 and Proposition 5 means that all second preimage resistant polynomials must have genus at least three.

As outlined above, elliptic (genus one) and hyperelliptic (genus two) polynomials are not second preimage resistant because there are non-trivial automorphisms on the associated curves. We say that a polynomial $f \in \mathbb{Z}[x, y]$ is automorphism free if, for all but finitely many $c \in \mathbb{Q}$, the curve $f(x, y)=c$ has no automorphisms over $\mathbb{Q}$, apart from the trivial map $(x, y) \mapsto(x, y)$. It is natural to conjecture that every automorphism-free polynomial $f \in \mathbb{Z}[x, y]$ is second preimage resistant.

Poonen constructs a large family of automorphism-free polynomials, in arbitrarily many variables and of arbitrarily large degree [29]. For example, he proves that the polynomial $f(x, y)=x^{3}+x y^{3}+y^{4}$ is automorphism-free over the rationals $[29]$.

A Historical Aside: $q$-Way Preimage Resistance. A stronger notion of preimage resistance for a function $f: U \rightarrow V$, called $q$-way preimage resistance, states that given a random $v \in V$ and random points $u_{1}, \ldots, u_{q}$ in $U$ such that $v=f\left(u_{1}\right)=\cdots=f\left(u_{q}\right)$, it is difficult to find a new point $u \in U \backslash\left\{u_{1}, \ldots, u_{q}\right\}$ such that $f(u)=v$.

As before, one can define a similar property for polynomials. That is, a polynomial $f$ in $\mathbb{Z}[x, y]$ is $q$-way preimage resistant if, for a random RSA moduli $N$ and a random $c \in \mathbb{Z}_{N}$, given $q$ points on the curve $f(x, y)=c$ in $\mathbb{Z}_{N}$, it is hard to find another point on this curve.

Kilian and Petrank 19] proposed an authentication scheme whose security is based on the $q$-way preimage resistance of the polynomial $f_{\mathrm{KP}}(x, y)=x^{e}-y^{e}$, for some small odd $e$, say $e=17$. In their scheme, $q$ is the total number of users in the system. Naor [25] refers to the computational assumption that $f_{\mathrm{KP}}$ is $q$-way preimage resistant as the Difference RSA Assumption. We note that the polynomial $f_{\mathrm{KP}}$ is not even second preimage resistant because there is a non-trivial automorphism $(x, y) \mapsto(-y,-x)$ on the curve. In other words, for any point $\left(x_{0}, y_{0}\right)$ we have that $f_{\mathrm{KP}}\left(x_{0}, y_{0}\right)=f_{\mathrm{KP}}\left(-y_{0},-x_{0}\right)$. This bad symmetry appears to violate the security properties needed for the Kilian-Petrank identification scheme, but the scheme can be modified to resist such attacks.

Camenisch and Stadler [10, Sec. 6] used a similar assumption to construct group signatures. They need the polynomial $f_{\mathrm{CS}}(x, y)=x^{e_{1}}+a y^{e_{2}}$ to be $q$-way preimage resistant for some small $e_{1}$ and $e_{2}$. They propose using $e_{1}=5$ and 
$e_{2}=3$. We observe in that next section that the polynomial $f(x, y)=x^{5}+y^{3}$ is not collision resistant. Nevertheless, it may be second preimage resistant.

\subsection{Collision-Resistant Polynomials}

A function $f: U \rightarrow V$ is collision resistant if it is difficult to find a pair $u \neq u^{\prime} \in$ $U$ such that $f(u)=f\left(u^{\prime}\right)$. We define a similar notion for polynomials:

Definition 7. For a polynomial $f$ in $\mathbb{Z}\left[x_{1}, \ldots, x_{\ell}\right]$ and an integer $N$, we say that $\bar{x}, \bar{y} \in\left(\mathbb{Z}_{N}\right)^{\ell}$ are an $N$-collision for $f$ if $f(\bar{x})=f(\bar{y})$ in $\mathbb{Z}_{N}$ and $\bar{x} \neq \bar{y}$.

Definition 8. A polynomial $f$ in $\mathbb{Z}\left[x_{1}, \ldots, x_{\ell}\right]$ is collision resistant if for every p.p.t. algorithm $\mathcal{A}$ the following advantage is a negligible function of $\lambda$ :

$$
\operatorname{Adv}_{\mathcal{A}, f}(\lambda):=\operatorname{Pr}[N \leftarrow \operatorname{RSAgen}(\lambda): \mathcal{A}(N) \text { is an } N \text {-collision for } f] .
$$

In the previous two subsections, we observed that polynomials $f \in \mathbb{Z}[x, y]$ which are of genus $g \leq 2$ or which are hyperelliptic, are not second preimage resistant and thus are not collision resistant.

Even polynomials that are second preimage resistant are not necessarily collision resistant. For example, in Section 3.2 we suggested that the polynomial $f(x, y)=x^{3}+x y^{3}+y^{4}$ may be second preimage resistant. However, it is certainly not collision resistant, since for any $r \in \mathbb{Q}$, the points $\left(r^{4}, 0\right)$ and $\left(0, r^{3}\right)$ constitute a collision.

Attacking Collision Resistance over the Rationals. Suppose that a polynomial $f \in \mathbb{Z}\left[x_{1}, \ldots, x_{\ell}\right]$ has a rational collision. That is, there are rational points $\bar{x}_{0} \neq \bar{x}_{1}$ in $\mathbb{Q}^{\ell}$ such that $f\left(\bar{x}_{0}\right)=f\left(\bar{x}_{1}\right)$. Then, for most 1 RSA moduli $N$, the points $\bar{x}_{0}$ and $\bar{x}_{1}$ give a collision for $f$ in $\mathbb{Z}_{N}$. This breaks the collision resistance of $f$ when the security parameter $\lambda$ is sufficiently large. Indeed, for sufficiently large $\lambda$ the attack algorithm can construct the fixed rational points $\bar{x}_{0}$ and $\bar{x}_{1}$ by exhaustive search and obtain collisions for $f$ for most RSA moduli output by $\operatorname{RSAgen}(\lambda)$.

The discussion above shows that if a polynomial $f \in \mathbb{Z}\left[x_{1}, \ldots, x_{\ell}\right]$ has a rational collision then $f$ is not collision resistant. We summarize this in the following proposition.

Proposition 9. If a polynomial $f \in \mathbb{Z}\left[x_{1}, \ldots, x_{\ell}\right]$ is collision resistant then the function $f: \mathbb{Q}^{\ell} \rightarrow \mathbb{Q}$ must be injective.

If $f \in \mathbb{Z}\left[x_{1}, \ldots, x_{\ell}\right]$ defines an injective function from $\mathbb{Q}^{\ell}$ to $\mathbb{Q}$ then $f$ is said to be an injective polynomial. Proposition 9 shows that the search for collisionresistant polynomials must begin with the search for an injective polynomial over the rationals.

${ }^{1}$ The points $\bar{x}_{0}$ and $\bar{x}_{1}$ give a collision in $\mathbb{Z}_{N}$ whenever $N$ is relatively prime to their denominators and $\overline{x_{0}} \neq \overline{x_{1}} \bmod N$. This holds with overwhelming probability for sufficiently large $\lambda$. 
Injective Polynomials. Even the existence of bivariate injective polynomials is currently an open problem. Poonen [30] shows that they exist under certain number theoretic conjectures. Moreover, Poonen [30, Lemma 2.3] shows that if $f \in \mathbb{Z}[x, y]$ has only a finite number of rational collisions then one can use $f$ to construct an injective polynomial $g \in \mathbb{Z}[x, y]$ by pre-composing $f$ with a suitable polynomial map. In other words, an "almost" injective polynomial can be converted to an injective one.

Although proving that a particular polynomial is injective over $\mathbb{Q}$ is currently out of reach, there are simple polynomials that appear to have this property. In particular, Don Zagien 2 conjectures that the polynomial $f_{\text {zAG }}(x, y):=x^{7}+$ $3 y^{7}$ (the "Zagier polynomial") defines an injective function from $\mathbb{Q}^{2}$ to $\mathbb{Q}$. As indirect evidence, Cornelissen [13, Remarque 10] and Poonen [30, Remark 1.7] remark that the four-variate generalization of the $a b c$-conjecture 7 implies that $f(x, y)=x^{e}+3 y^{e}$ is injective over the rationals for "sufficiently large" odd integers $e$. Experimentally, we have confirmed that there are no rational collisions in $f_{\mathrm{zAG}}$ for rationals with height less than 100 .

$\ell$-Variate Injective Polynomials over $\mathbb{Q}$ from Merkle-Damgård. Given a bivariate injective polynomial over $\mathbb{Q}$, it is possible to construct $\ell$-variate injective polynomials over $\mathbb{Q}$ for every $\ell>2$ using the Merkle-Damgård construction for collision-resistant hash functions [15122]. For example, applying one step of Merkle-Damgård to $f_{\mathrm{ZAG}}$ shows that if $f_{\mathrm{ZAG}}$ is injective then so is the following three-variate polynomial:

$$
g(x, y, z)=\left(x^{7}+3 y^{7}\right)^{7}+3 z^{7} .
$$

Injective Polynomials and Collision Resistance. Proposition 9 states that, for a polynomial $f$ to be collision resistant over $\mathbb{Z}_{N}, f$ must be injective over the rationals. The following conjecture asserts the converse: injectivity over the rationals is sufficient for collision resistance.

Conjecture 10. If $f \in \mathbb{Z}\left[x_{1}, \ldots, x_{\ell}\right]$ is injective over $\mathbb{Q}$ then $f$ is collision resistant.

This conjecture is based on the intuition that the only efficient way to find collisions in $f$ over $\mathbb{Z}_{N}$ is to find collisions in $f$ over $\mathbb{Q}$. Since collisions over $\mathbb{Q}$ do not exist it may be difficult to find collisions over $\mathbb{Z}_{N}$.

We only state Conjecture 10 to stimulate further research on this topic. The conjecture is not needed for this paper. For the applications described in this paper, we only need the collision resistance of an explicit low-degree polynomial in $\mathbb{Z}[x, y]$. Nevertheless, if Conjecture 10 is true it would give a clean characterization of collision resistant polynomials in terms of their arithmetic properties.

For the applications in paper, the following assumption suffices.

${ }^{2}$ Gunther Corneliseen attributes to Don Zagier the suggestion that $f(x, y)=x^{7}+3 y^{7}$ is collision-free over the rationals [13, Remarque 10]. 
Assumption 11. The Zagier polynomial $f_{\mathrm{ZAG}}(x, y)=x^{7}+3 y^{7} \in \mathbb{Z}[x, y]$ is collision resistant.

We see that breaking Assumption 11 would either: (a) resolve a 15-year open number theoretic problem by showing that $f_{\mathrm{zAG}}$ is non-injective, or (b) find $\mathbb{Z}_{N}$ collisions that are not rational collisions. We next review two potential avenues for attacks of type (b) and discuss why they do not apply.

Attack Strategy I: Related Non-injective Polynomials over $\mathbb{Q}$. One potential avenue for attacking the collision resistance of $f_{\mathrm{ZAG}}$ in $\mathbb{Z}_{N}$ is to look for a polynomial $h \in \mathbb{Z}[x, y]$ such that

$$
g(x, y):=f(x, y)+N \cdot h(x, y)
$$

is not injective over $\mathbb{Q}$. If $\left(x_{0}, y_{0}\right)$ and $\left(x_{1}, y_{1}\right)$ in $\mathbb{Q}^{2}$ are a rational collision for $g$ then by reducing this pair modulo $N$ we may ${ }^{3}$ obtain a $\mathbb{Z}_{N}$ collision for $f(x, y)$. We say that $h$ is "useful" if there exists a rational collision for $g(x, y)$ that gives a $\mathbb{Z}_{N}$ collision for $f(x, y)$. It is easy to show that there are many useful polynomials $h$ : every $\mathbb{Z}_{N}$ collision for $f(x, y)$ gives a useful polynomial $h$. However, we do not know how to construct a useful $h$ just given $f$ and $N$. Furthermore, even if efficiently constructing a useful $h$ is possible, the attack algorithm will need to find a rational collision on the resulting $g$ and this may not be feasible in polynomial time.

Attack Strategy II: Algebraic Extensions. Another avenue for attacking the collision resistance of $f_{\mathrm{ZAG}}$ in $\mathbb{Z}_{N}$ is via algebraic extensions. Let $g$ be an irreducible polynomial in $\mathbb{Z}[x]$ and consider the number field $\mathbb{K}=\mathbb{Q}[x] /(g)$. Suppose the adversary constructs $g$ so that it knows an efficiently computable $\operatorname{map} \rho: \mathbb{K} \rightarrow \mathbb{Z}_{N}$ (this can be done by choosing the polynomial $g$ so that the adversary knows a zero of $g$ in $\mathbb{Z}_{N}$ ). Now, even if $f_{\text {zAG }}$ is injective as a function $\mathbb{Q}^{2} \rightarrow \mathbb{Q}$, it may not be injective as a function $\mathbb{K}^{2} \rightarrow \mathbb{K}$. For example, $f_{\text {ZAG }}$ is not injective over the extension $\mathbb{K}=\mathbb{Q}[\sqrt[7]{3}]$ : the points $(\sqrt[7]{3}, 0)$ and $(0,1)$ are a collision. If the adversary could find a collision of $f_{\mathrm{ZAG}}$ in $\mathbb{K}^{2}$ this collision may lead to a $\mathbb{Z}_{N}$ collision for $f_{\text {zAG }}$. However, for a random RSA modulus $N$, it is not known how to efficiently construct an extension $\mathbb{K}$ such that (i) $f_{\text {ZAG }}: \mathbb{K}^{2} \rightarrow \mathbb{K}$ is not injective, and (ii) the adversary has an efficiently computable map $\rho: \mathbb{K} \rightarrow \mathbb{Z}_{N}$.

Assumption 11 merits further analysis and we hope that this work will stimulate further research on this question.

Non-collision Resistant Polynomials. Simple variations of Zagier's polynomial are trivially not injective and therefore not collision resistant. For example, the polynomials

$$
f_{1}(x, y)=x^{7}+y^{7} \quad \text { and } \quad f_{2}(x, y)=x^{7}+2 y^{7}
$$

${ }^{3}$ If $\left(x_{0}, y_{0}\right)$ and $\left(x_{1}, y_{1}\right)$ happens to reduce to the same point modulo $N$ or if one of the denominators is not relatively prime to $N$ then this rational collision for $g$ does not give a $\mathbb{Z}_{N}$ collision for $f$. 
in $\mathbb{Z}[x, y]$ are not collision resistant. The polynomial $f_{1}$ is not injective because for all $x_{0} \neq y_{0}$ in $\mathbb{Z}$ the points $\left(x_{0}, y_{0}\right)$ and $\left(y_{0}, x_{0}\right)$ are a collision for $f_{1}$. The polynomial $f_{2}$ is not collision resistant because for all $t \neq 0$ in $\mathbb{Z}$ the points $(-t, 0)$ and $(t,-t)$ are a collision for $f_{2}$.

Similarly, polynomials of the form $f(x, y)=x^{e_{1}}+b y^{e_{2}} \in \mathbb{Z}[x, y]$ for some $b \in \mathbb{Z}$ where $\operatorname{gcd}\left(e_{1}, e_{2}\right)=1$ are not injective and therefore not collision resistant. To see why observe that if the equation $\alpha e_{1}-\beta e_{2}=1$ has integer solutions $\left(\alpha_{0}, \beta_{0}\right)$ and $\left(\alpha_{1}, \beta_{1}\right)$ then $\left(t^{\alpha_{0}}, t^{\beta_{1}}\right)$ and $\left(t^{\alpha_{1}}, t^{\beta_{0}}\right)$ are a collision for $f$.

Random Self-reduction. Finally, we mention that the collision finding problem for the family of polynomials $\left\{x^{e}+a y^{e}\right\}_{a \in \mathbb{Z}_{N}}$ has a random self reduction. Given a collision-finding algorithm $\mathcal{A}(N, a)$ that outputs a $\mathbb{Z}_{N}$ collision in $x^{e}+a y^{e}$ for a non-negligible fraction of choices of $a \in \mathbb{Z}_{N}$, it is possible to construct a collision-finding algorithm $\mathcal{B}(N, a)$ that finds collisions for every choice of $a$ with high probability. On input $(N, a)$ Algorithm $\mathcal{B}$ chooses a random $r \leftarrow \mathbb{Z}_{N}$, and calls $\mathcal{A}\left(N, r^{e} a\right)$. When $\mathcal{A}$ outputs the collision $\left(x_{0}, y_{0}\right),\left(x_{1}, y_{1}\right)$, algorithm $\mathcal{B}$ obtains the following collision on the original curve: $\left(x_{0}, r y_{0}\right),\left(x_{1}, r y_{1}\right)$. If $\mathcal{A}$ fails then $\mathcal{B}$ can try again with a fresh random choice of $a \in \mathbb{Z}_{N}$. After an expected polynomial number of iterations algorithm $\mathcal{B}$ will find a collision for the given polynomial $x^{e}+a y^{e}$.

\section{A Nestable Commitment Scheme from Polynomials over $\mathbb{Z}_{N}$}

Having argued that it is infeasible to find collisions in the function $f_{\mathrm{ZAG}}(x, y)=$ $x^{7}+3 y^{7} \bmod N$ (Assumption 11), we now turn to the cryptographic applications of this new computational assumption. In this section, we demonstrate that the collision-resistance of $f_{\text {ZAG }}$ leads to a commitment scheme where the procedure for verifying that a commitment was opened correctly uses only low-degree polynomials. The new commitment scheme is statistically hiding and its computational binding property is based on Assumption 11.

The commitment scheme composes naturally with zero-knowledge proofs of knowledge involving Pedersen commitments. In particular, given a Pedersen commitment $C$ to one of our low-degree commitments, there is a succinct zeroknowledge protocol which proves knowledge of an opening of an opening of $C$. We call the inner commitment scheme nestable, since it can be efficiently nested inside of a Pedersen commitment. We discuss applications of nestable commitments in Sections 4.4 and 5 .

\subsection{Commitments}

A commitment scheme is a tuple of efficient algorithms (Setup, Commit, Open), with the following functionalities:

$\operatorname{Setup}(\lambda) \rightarrow$ pp. The Setup routine is a randomized algorithm that runs in time polynomial $\lambda$ and returns public parameters pp. These parameters define 
a message space $\mathcal{M}$, a space of random blinding values $\mathcal{R}$, and a space of commitments $\mathcal{C}$. The following algorithms take the public parameters pp as an implicit argument.

Commit $(m) \rightarrow(c, r)$. Given a message $m \in \mathcal{M}$, return a commitment $c \in \mathcal{C}$ and a random blinding value $r \in \mathcal{R}$ used to open the commitment.

Open $(c, m, r) \rightarrow\{0,1\}$. Given a commitment $c$, a message $m$, and a blinding value $r$, return " 1 " if $(m, r)$ is a valid opening of $c$ and " 0 " otherwise.

For correctness, we require that, for all $m \in \mathcal{M}$ :

$$
\operatorname{Pr}[\mathrm{pp} \leftarrow \operatorname{Setup}(\lambda) ;(c, r) \leftarrow \operatorname{Commit}(m): \operatorname{Open}(c, m, r)=1] \geq 1-\operatorname{negl}(\lambda) .
$$

A statistically hiding commitment scheme must satisfy two security properties:

- Statistically Hiding. For any two messages $m_{0}$ and $m_{1}$ in $\mathcal{M}$, a commitment to $m_{0}$ is statistically indistinguishable from a commitment to $m_{1}$.

- Computationally Binding. For any p.p.t. adversary $\mathcal{A}$, the adversary has negligible advantage in producing two different valid openings of the same commitment. More precisely,

$$
\begin{aligned}
& \operatorname{Pr}\left[\mathrm{pp} \leftarrow \operatorname{Setup}(\lambda) ;\left(c, m, r, m^{\prime}, r^{\prime}\right) \leftarrow \mathcal{A}(\mathrm{pp}):\right. \\
& \left.\quad \text { Open }(c, m, r)=1 \wedge \operatorname{Open}\left(c, m^{\prime}, r^{\prime}\right)=1 \wedge(m, r) \neq\left(m^{\prime}, r^{\prime}\right)\right] \leq \operatorname{negl}(\lambda) .
\end{aligned}
$$

\subsection{Construction}

The public parameters for our new commitment scheme consist only of an RSA modulus $N$, for which no one knows the factorization. To commit to a value $m \in$ $\mathbb{Z}_{N}^{*}$, the committer samples a random blinding value $r$ from $\mathbb{Z}_{N}^{*}$ and computes the value of $f_{\mathrm{ZAG}}$ at the point $(m, r)$.

The construction of the new commitment scheme follows.

$\operatorname{Setup}(\lambda) \rightarrow N$. The value $N$ is an RSA modulus - the product of two random len $(\lambda)$-bit primes $p$ and $q$ such that $\operatorname{gcd}(p-1, q-1,7)=1$. The commitment space $\mathcal{C}$ is $\mathbb{Z}_{N}$. The message space $\mathcal{M}$ and the space of blinding values $\mathcal{R}$ are $\mathbb{Z}_{N}^{*}$

Commit $(m) \rightarrow(c, r)$. Choose a random blinding value $r \leftarrow \mathbb{Z}_{N}^{*}$ and set $c \leftarrow$ $m^{7}+3 r^{7}$ in $\mathbb{Z}_{N}$. Return $r$ as the commitment secret.

Open $(c, m, r) \rightarrow\{0,1\}$. Output " 1 " if $m, r \in \mathbb{Z}_{N}^{*}$ and if $c=m^{7}+3 r^{7}$ in $\mathbb{Z}_{N}$. Output " 0 " otherwise.

Security Properties. The following theorem summarizes the security properties of the scheme.

Theorem 12. The commitment scheme is statistically hiding and computationally binding under Assumption 11.

Proof. Statistical hiding follows from a standard argument given in Appendix A Computational binding follows directly from the collision resistance of $f_{\mathrm{ZAG}}$ over $\mathbb{Z}_{N}$. One issue is Setup algorithm generates a random $N$ such that $\operatorname{gcd}(\phi(N), 7)=$ 
1 whereas Assumption 11 imposes no such restriction on $N$. Nevertheless, Assumption 11 implies the collision resistance of $f_{\mathrm{ZAG}}$ for this modified distribution of $N$ : By way of contradiction, assume there were an algorithm $\mathcal{A}$ which finds collisions in $f_{\mathrm{ZAG}}$ with non-negligible probability $\epsilon$ when $\operatorname{gcd}(\phi(N), 7)=1$. Since algorithm RSAgen in Assumption 11 generates such $N$ with probability about $(5 / 6)^{2}=25 / 36$ it follows that $\mathcal{A}$ will find collisions in with probability at least $(25 / 36) \epsilon$ when $N$ is sampled as in algorithm RSAgen, violating Assumption 11 .

Efficiency. Generating and verifying standard Pedersen commitments requires two modular exponentiations (or elliptic curve scalar multiplications). In contrast, our scheme requires only a few modular multiplications. On a workstation with a $3.20 \mathrm{GHz}$ processor, for example, computing 10,000 Pedersen commitments in a subgroup of order $\approx 2^{256}$ modulo a 2048 -bit prime takes 16.54 seconds. Computing the same number of commitments using this new scheme takes 0.925 seconds - a factor of $17.9 \times$ speed-up.

\subsection{Nestable Commitments}

We say that a commitment scheme (Setup, Commit, Open) is nestable if, given Pedersen commitments to a message $m$, randomness $r$, and a commitment $c$, there is an succinct zero-knowledge proof of knowledge of values $m, r$, and $c$, such that $c=$ Commit $(m, r)$. In other words, there is a succinct protocol for proving knowledge of an opening of an opening of a Pedersen commitment. For our purposes, a succinct zero-knowledge protocol is one in which proof length is $k|c|$ bits long, where $k$ is a constant which does not depend on the security parameter.

We adopt the notation of Camenisch and Stadler [9] for specifying zeroknowledge proof-of-knowledge protocols. For example, $\operatorname{PoK}\left\{x, y: X=g^{x} \vee Y=\right.$ $\left.g^{x}\right\}$ indicates a protocol in which the prover and verifier share public values $g$, $X$, and $Y$, and the prover demonstrates knowledge of either a value $x$ such that $X=g^{x}$ or a value $y$ such that $Y=g^{y}$.

Given Pedersen commitments

$$
C_{m}=g^{m} h^{s_{m}} \quad C_{r}=g^{r} h^{s_{r}} \quad C_{c}=g^{c} h^{s_{c}}
$$

a nestable commitment scheme has a succinct zero-knowledge protocol which proves knowledge of the statement:

$$
\operatorname{PoK}\left\{m, r, c, s_{m}, s_{r}, s_{c}: C_{m}=g^{m} h^{s_{m}} \wedge C_{r}=g^{r} h^{s_{r}} \wedge C_{c}=g^{\operatorname{Commit}(m, r)} h^{s_{c}}\right\} .
$$

For the commitment scheme outlined above, Commit $(m, r)=m^{7}+3 r^{7} \bmod N$, so the proof of knowledge protocol is:

$$
\operatorname{PoK}\left\{m, r, c, s_{m}, s_{r}, s_{c}: C_{m}=g^{m} h^{s_{m}} \wedge C_{r}=g^{r} h^{s_{r}} \wedge C_{c}=g^{m^{7}+3 r^{7}} h^{s_{c}}\right\} .
$$

The group $\mathbb{G}=\langle g\rangle=\langle h\rangle$ used for the proof must be a group of composite order $N$, where $N$ is the RSA modulus used in the commitment scheme. As usual for 
Pedersen commitments, no one should know the discrete $\operatorname{logarithm}_{\log _{g}} h$ in $\mathbb{G}$. For example, $\mathbb{G}$ might be the order- $N$ subgroup of the group $\mathbb{Z}_{p}^{*}$ for a prime $p=2 k N+1$, where $k$ is a small prime. Alternatively, $\mathbb{G}$ could be an elliptic curve group of order $N$.

The fact that the verification equation for our commitment scheme is a fixed low-degree polynomial means that this proof can be executed succinctly using standard techniques [10]. This proof requires only one challenge and 20 elements of $\mathbb{G}$. If $N$ is a 2048-bit modulus, then the proof is roughly $5 \mathrm{~KB}$ in length.

In contrast, nesting Pedersen commitments inside of other Pedersen commitments does not lead to succinct proofs of knowledge. The shortest proofs of knowledge for nested Pedersen commitments require a number of group elements that is linear in the security parameter [11, Sec. 5.3.3], whereas our proof requires only a constant number of group elements.

Being able to prove knowledge of an opening of a commitment which is itself nested inside of a commitment proves useful in constructing distributed e-cash schemes (Section 4.4) and set membership proofs (Section 5).

\subsection{Application Sketch: Anonymous Bitcoins}

The Zerocoin scheme for anonymizing Bitcoin transactions requires a proof of knowledge of an opening of an opening of a commitment 24. For this purpose, Zerocoin uses Pedersen commitments nested inside of Pedersen commitments, which requires a proof-of-knowledge of the form: $\operatorname{PoK}\left\{m, r, s: \hat{c}=\hat{g}^{\left(g^{m} h^{r}\right)} \hat{h}^{s}\right\}$. The number of group elements exchanged in this proof is linear in the security parameter, since the proof uses single-bit challenges.

By using our nestable commitment scheme for the "inner" commitment, we reduce the number of group elements from linear to constant in the security parameter. This reduces the length of anonymous coin transactions in the Zerocoin scheme by roughly $70 \%$ (down to $12.0 \mathrm{KiB}$ from $39.4 \mathrm{KiB}$ when using a 2048-bit RSA modulus). When instantiated with our nestable commitments, Zerocoin maintains its unconditional privacy property and maintains doublespending prevention under Assumption 11.

\section{Succinct Set Membership Proofs}

A cryptographic accumulator, first defined by Benaloh and De Mare [5], is a primitive which allows a prover to accumulate large set of values $S=\left\{x_{1}, \ldots, x_{n}\right\}$ into a single short value $A$. For every value $x_{i}$ in the accumulator, there is an accompanying short witness $w_{i}$. By exhibiting a valid $\left(x_{i}, w_{i}\right)$ pair, a prover can convince a verifier that the value $x_{i}$ was actually accumulated into $A$. Informally, the security property of the accumulator requires that it be difficult to find a valid value-witness pair $\left(x^{*}, w^{*}\right)$ such that $x^{*} \notin S$.

Benaloh and De Mare give one example application of this primitive: the administrator of a club can accumulate the names of the members of the club into an accumulator $A$, distribute a witness to each member, and publish the accumulator value $A$. The value $A$ is a concise representation of the club's membership 
list. A person can prove membership in the club by revealing her name $x_{i}$ and the witness $w_{i}$ to a verifier.

Camenisch-Lysyanskaya extend the basic accumulator primitive to allow for zero-knowledge proofs of accumulator membership [8]. That is, a prover can convince a verifier that the prover "knows" a valid value-witness pair $(x, w)$ for a particular accumulator $A$, without revealing $x$ or $w$. This augmented primitive allows for privacy-preserving authentication: a club member can prove that she is some member of the club defined by a membership list $A$ without revealing which member she is.

We provide a construction that offers the same functionality as the CamenischLysyanskaya scheme with the cost of requiring slightly larger proofs - of length $O(\log |S|)$ instead of length $O(1)$. The benefit of our construction is its simplicity: compared with the Camenisch-Lysyanskaya proof, which requires a nuanced security analysis, ours is relatively straightforward.

\subsection{Definitions}

A cryptographic accumulator is a tuple of algorithms (Setup, Accumulate, Witness, Verify) with the following functionalities:

$\operatorname{Setup}(\lambda) \rightarrow$ pp. Given a security parameter $\lambda$ as input, output the public parameters pp. The other functions take pp as an implicit input. Setup runs in time polynomial in $\lambda$.

Accumulate $\left(S=\left\{x_{1}, \ldots, x_{n}\right\}\right) \rightarrow A$. Accumulate the $n$ items in the set $S$ into an accumulator value $A$.

Witness $(S, x) \rightarrow w$ or $\perp$. If $x \notin S$, return $\perp$. Otherwise, return a witness $w$ that $x$ was accumulated in $\operatorname{Accumulate}(S)$. To be useful, the length of $w$ should be short (constant or logarithmic) in the size of $S$.

$\operatorname{Verify}(A, x, w) \rightarrow\{0,1\}$. Return " 1 " if the value-witness pair $(x, w)$ is valid for the accumulator $A$. Return "0" otherwise.

Camenisch and Lysyanskaya, following Barić and Pfitzmann [4], define an accumulator as secure, if for all polynomial-time adversaries $\mathcal{A}$ :

$$
\begin{aligned}
\operatorname{Pr}[\mathrm{pp} \leftarrow \operatorname{Setup}(\lambda) ; & \left(S, x^{*}, w^{*}\right) \leftarrow \mathcal{A}(\mathrm{pp}) ; x^{*} \notin S ; \\
& \left.A \leftarrow \operatorname{Accumulate}(S): \operatorname{Verify}\left(A, x^{*}, w^{*}\right)=1\right] \leq \operatorname{negl}(\lambda) .
\end{aligned}
$$

If an accumulator satisfies this definition, then it is infeasible for an adversary to prove that a value $x$ was accumulated in a value $A$ if it was not.

Zero-Knowledge Proof of Knowledge of an Accumulated Value. In many applications, it is useful for a prover to be able to convince a verifier that the prover knows some value inside of an accumulator without revealing which value the prover knows. Such a proof protocol should satisfy the standard properties of soundness, completeness, and zero-knowledgeness [11, Sec. 2.9]. Camenisch and Lysyanskaya construct one such proof-of-knowledge protocol for the strong-RSA accumulator [8] and we exhibit a protocol for a Merkle-tree-style accumulator in Section 5.3 . 


\subsection{Construction}

Given a collision-resistant hash function $H: D \times D \rightarrow D$, which operates on a domain $D$ such that $S \subseteq D$, it is possible to construct a simple accumulator using Merkle trees. For example, given a set $S=\left\{x_{1}, x_{2}, x_{3}, x_{4}\right\}$, the accumulator value $A$ is the value $A \leftarrow H\left(H\left(x_{1}, x_{2}\right), H\left(x_{3}, x_{4}\right)\right)$. A witness $w_{i}$ that an element $x_{i}$ is in the accumulator is the set of $O(\log |S|)$ nodes along the Merkle tree needed to verify a path from $x_{i}$ to the root (labeled $A$ ).

The limitation of this accumulator construction is that it no longer admits simple zero-knowledge proofs of knowledge of $(x, w)$ pairs, unless $H$ has a very special form. For instance, if $H$ is a standard cryptographic hash function (e.g., SHA-256), there is no straightforward zero-knowledge protocol for proving knowledge in zero knowledge of a preimage under $H$. By instantiating $H$ with the function $H(x, y)=x^{7}+3 r^{7} \bmod N$, as we demonstrate in the following section, it is possible to execute this zero-knowledge proof succinctly.

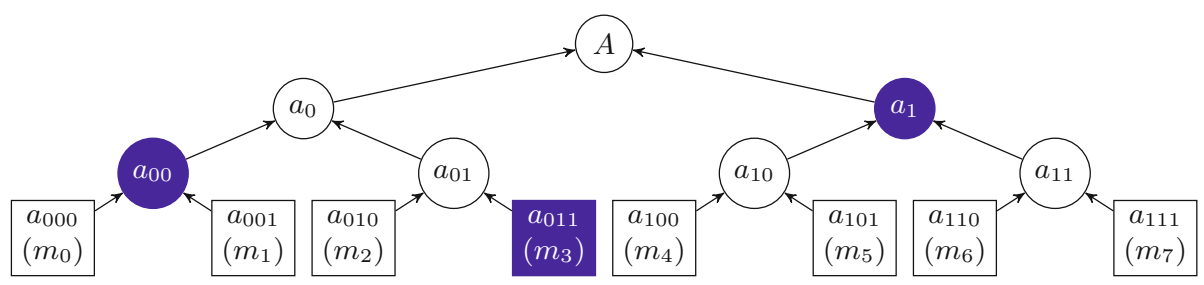

Fig. 1. A perfect Merkle tree with eight leaves rooted at $A$. The shaded nodes are a witness to the fact that $m_{2}$ is accumulated in $A$. The tree invariant is $a_{i}=H\left(a_{i 0}, a_{i 1}\right)$.

We first recall the standard construction of Merkle trees 21] and then describe the zero-knowledge proof construction. The construction from a general collisionresistant hash function family $\left\{\mathcal{H}_{\lambda}\right\}_{\lambda=1}^{\infty}$ follows.

$\operatorname{Setup}(\lambda) \rightarrow H$. Given a security parameter $\lambda$ as input, sample a $\lambda$-secure collision-resistant hash function $H$ from $\mathcal{H}_{\lambda}$. Setup runs in time polynomial in $\lambda$.

Accumulate $\left(S=\left\{x_{1}, \ldots, x_{n}\right\}\right) \rightarrow A$. If $|S|$ is not a power of two, insert "dummy" elements into $S$ (e.g., by duplicating the first element of $S$ ) until $|S|$ is a power of two. Construct a perfect Merkle tree of depth $d=\log _{2}|S|$ using the hash function $H$ with the members of $S$ as its leaves and return the root $A$. Figure 1 depicts an example tree of depth three.

Witness $(S, x) \rightarrow w$ or $\perp$. If $x \notin S$, return $\perp$. Otherwise, let the path from $A$ to the message $x$ be: $P=\left(A, a_{b_{1}}, a_{b_{1} b_{2}}, a_{b_{1} b_{2} b_{3}}, \ldots, a_{b_{1} \ldots b_{d}}\right)$, where $a_{i 0}$ is the left child of node $a_{i}, a_{i 1}$ is the right child of node $a_{i}$, and $d$ is the number of edges between the root and leaf labeled $x$ in the tree. The first component of the witness is the list of siblings of the nodes in the path $P: w_{\alpha}=$ $\left(a_{\bar{b}_{1}}, a_{b_{1} \bar{b}_{2}}, a_{b_{1} b_{2} \bar{b}_{3}}, \ldots, a_{b_{1} \ldots \bar{b}_{d}}\right)$. The second component of the witness is a bit 
vector indicating where $x$ is located in the tree: $w_{\beta}=\left(b_{1}, b_{2}, \ldots, b_{d-1}, b_{d}\right)$. The witness is $w=\left(w_{\alpha}, w_{\beta}\right)$.

$\operatorname{Verify}(A, x, w) \rightarrow\{0,1\}$. Interpret the witness as $\left(w_{\alpha}, w_{\beta}\right)$ such that $w_{\alpha}=$ $\left(w_{1}, \ldots, w_{d}\right)$ and $w_{\beta}=\left(b_{1}, \ldots, b_{d}\right)$. To verify the witness, let $t_{d}=x$ and recompute the intermediate nodes of the tree from the leaf back to the root. Specifically, compute test nodes $t_{i}$ for $i=d-1, \ldots, 0$ :

$$
t_{i}=\left\{\begin{array}{l}
H\left(t_{i+1}, w_{i+1}\right): \text { if } b_{i}=0 \\
H\left(w_{i+1}, t_{i+1}\right): \text { if } b_{i}=1
\end{array}\right.
$$

Return " 1 " if $A=t_{0}$ and " 0 " otherwise.

\subsection{Proof of Knowledge of an Accumulated Value}

When instantiated with a general hash function $H$, the Merkle-tree accumulator of the prior section does not admit a succinct proof of knowledge of an accumulated value. When instantiated with our new hash function $H(x, y)=$ $x^{7}+3 y^{7} \bmod N$, however, there is a succinct proof of knowledge that the prover knows an opening of a Pedersen commitment $C_{m}$ such that some leaf of the accumulator Merkle tree has label $m$. The proof requires a group $\mathbb{G}=\langle g\rangle=\langle h\rangle$ of order $N$, as in Section 4.3. The proof length is $\log |S|$, for a set $S$ of elements accumulated.

The Setup algorithm outputs an RSA modulus $N \leftarrow \operatorname{RSAgen}(\lambda)$ such that $\operatorname{gcd}(\phi(N), 7)=1$ and such that no one knows the factorization of $N$. The hash function $H$ is $H(x, y)=x^{7}+3 y^{7} \bmod N$ and the accumulator domain $D$ is $\mathbb{Z}_{N}^{*}$.

The high-level idea is that, if the prover wants to convince the verifier that a particular value $m$ is accumulated in $A$, the prover commits to the values of all of the nodes in the Merkle tree along the path from the root to the leaf labeled $m$. The prover also commits to all of the witness values needed to recreate the path from the leaf labeled $m$ down to the tree root. The prover can then convince the verifier in zero knowledge that these commitments together contain a path to some leaf in the tree, without revealing which one.

Assume that the prover has a value-witness pair $(x, w)$ which convinces a verifier that $x$ is accumulated in $A$. Denote the node values along the path from the root node, with value $A$, to the leaf node, with value $x$, in the Merkle tree as: $p=\left(p_{0}, p_{1}, \ldots, p_{d}\right)$. Note that $p_{0}=A$ and $p_{d}=x$.

The prover now commits to every value $p_{i}$ in this path and to the values of the left and right children of $p_{i}$ in the Merkle tree. If the value of the left child is $\ell_{i}$ and the right child is $r_{i}$, the commitments are, for $i=0, \ldots, d-1$ :

$$
P_{i}=g^{p_{i}} h^{s_{i}} \quad L_{i}=g^{\ell_{i}} h^{s_{i}^{\prime}} \quad R_{i}=g^{r_{i}} h^{s_{i}^{\prime \prime}}
$$

The prover opens $P_{0}$ by publishing $\left(p_{0}, s_{0}\right)$ and the verifier ensures that $p_{0}=A$ and that $P_{0}=g^{p_{0}} h^{s_{0}}$.

The prover now can prove, for $i=0, \ldots, d-1$, that each $\left(P_{i}, L_{i}, R_{i}\right)$ tuple is well-formed using a standard discrete logarithm proof:

$$
\mathrm{PoK}_{\alpha}\left\{\ell, r, s, s^{\prime}, s^{\prime \prime}: P_{i}=g^{\ell^{7}+3 r^{7}} h^{s} \wedge L_{i}=g^{\ell} h^{s^{\prime}} \wedge R_{i}=g^{r} h^{s^{\prime \prime}}\right\} .
$$


The prover then must prove that it knows an opening of the commitment $P_{i+1}$ such that the opening is equal to an opening of either $L_{i}$ or $R_{i}$. For $i=0, \ldots, d-$ 1, the prover proves:

$$
\mathrm{PoK}_{\beta}\left\{p, s, s_{\ell}, s_{r}: P_{i+1}=g^{p} h^{s} \wedge\left(L_{i}=g^{p} h^{s_{\ell}} \vee R_{i}=g^{p} h^{s_{r}}\right)\right\}
$$

The complete proof is the set of commitment pairs $\left\{\left(P_{i}, L_{i}, R_{i}\right)\right\}_{i=0}^{d}$, the $2 d$ proofs of knowledge, and the opening $\left(p_{0}, r_{0}\right)$ of the root commitment $P_{0}$. The total length is $O(d)=O(\log |S|)$, since the tree has depth $d=\log |S|$ and each of the elements of the proof has length which is constant in $|S|$.

Security. The completeness and zero-knowledgeness properties follows from the properties of the underlying zero-knowledge proofs used and from the fact that Pedersen commitments are perfectly hiding.

To show soundness, we must demonstrate that if the verifier accepts, it can extract a value-witness pair $\left(x^{*}, w^{*}\right)$ for the original Merkle tree with non-negligible probability by rewinding the prover. Starting at the root and working towards the leaves of the tree, we will be able to extract the prover's witness for each of the proofs of knowledge with non-negligible probability.

By induction on $i$, we can show that after $d$ steps, the verifier will be able to extract the value-witness pair $(x, w)$. The base case of the induction is $i=0$ and the verifier can extract a preimage of $A$ under $H$. From each of the $i \mathrm{PoK}_{\alpha} \mathrm{s}$, the verifier extracts an element of the witness $w_{\alpha}$ (the preimage of $p_{i}$ under $H$ ). From each of the $i \mathrm{PoK}_{\beta} \mathrm{s}$, the verifier extracts an element of the witness $w_{\beta}$ (whether the next node in the path is the left or right child of $p_{i}$ ).

\section{Claw-Free Functions, Signatures, and Chameleon Hashes}

In this section, we describe a few other applications arising from the assumed collision-freeness of the Zagier polynomial.

Claw-Free Functions and Signatures. Assumption 11 immediately gives rise to a family of trapdoor claw-free functions [14]. For each RSA modulus $N$ selected as in Section 4.2, we can define a function family:

$$
\mathcal{F}_{N}:=\left\{f_{a} \mid a \in \mathbb{Z}_{N}^{*}\right\} \quad \text { where } \quad f_{a}(x)=x^{7}+3 a^{7} \bmod N
$$

Following Damgård [14, a function family $\mathcal{F}_{N}$ is claw free if, given $\mathcal{F}_{N}$, it is difficult to find a "claw" $(x, y, a, b)$ such that $f_{a}(x)=f_{b}(y)$. For all p.p.t. adversaries $\mathcal{A}$, we require that:

$$
\operatorname{Pr}\left[N \leftarrow \operatorname{RSAgen}(\lambda),(x, y, a, b) \leftarrow \mathcal{A}(N): f_{a}(x)=f_{b}(y)\right] \leq \operatorname{negl}(\lambda)
$$

The claw-freeness of $\mathcal{F}_{N}$ follows from Assumption[11, since a claw in $\mathcal{F}_{N}$ implies a collision in $f(x, y)=x^{7}+3 y^{7} \bmod N$. Additionally, the function family $\mathcal{F}_{N}$ 
is trapdoor claw-free, since anyone with knowledge of the factors of $N$ can find claws easily by choosing $(x, y, a)$ arbitrarily and solving for $b$.

This family $\mathcal{F}_{N}$ is not quite a family of trapdoor claw-free permutations, since the range of two functions $f_{a}$ and $f_{b}$ in $\mathcal{F}_{N}$ are not necessarily equal (i.e., $f_{b}^{-1}\left(f_{a}(x)\right)$ is sometimes undefined). However, the fraction of choices of $(a, b, x)$ for which this event occurs is negligible, so it is possible to treat $\mathcal{F}_{N}$ as if it were a family of trapdoor claw-free permutations. In particular, this function family leads to a signature scheme secure against adaptive chosen message attacks in the standard model by way of the Goldwasser-Micali-Rivest signature construction [17].

Chameleon Hash. This commitment scheme immediately gives rise to a new chameleon hash function. A chameleon hash, as defined by Krawczyk and Rabin, is a public hash function $H(m, r)$ with a secret "trapdoor" [20. A chameleon hash function has three properties:

1. Without the trapdoor, it is difficult to find collisions in $H$. That is, it is hard to find colliding pairs $(m, r)$ and $\left(m^{\prime}, r^{\prime}\right)$ such that $H(m, r)=H\left(m^{\prime}, r^{\prime}\right)$.

2. Given the trapdoor, there is an efficient algorithm which takes $\left(m, r, m^{\prime}\right)$ as input and outputs a value $r^{\prime}$ such that $H(m, r)=H\left(m^{\prime}, r^{\prime}\right)$.

3. For any pair of messages $m$ and $m^{\prime}$ in the message space $\mathcal{M}$, the distributions $H(m, r)$ and $H\left(m^{\prime}, r^{\prime}\right)$ are statistically close if $r$ and $r^{\prime}$ are chosen at random.

Chameleon hashes are useful in building secure signature schemes in the standard model [16] and for a number of other applications [20].

To derive a chameleon hash scheme from our commitment scheme, set the public key to the RSA modulus $N$, and the secret key to the factorization of $N$. The hash function $H$ is then $H(m, r)=m^{7}+3 r^{7} \bmod N$. Without the factors of $N$, it is difficult to find collisions but anyone with knowledge of the factors of $N$ (the "trapdoor") can find collisions.

Chameleon hashes based on Pedersen commitments require two modular exponentiations to evaluate, while ours requires just a few modular multiplications.

\section{Conclusion and Future Work}

We have used arithmetic properties of bivariate polynomials over $\mathbb{Q}$ to reason about their cryptographic properties in the $\operatorname{ring} \mathbb{Z}_{N}$. Using one particular lowdegree polynomial, $f_{\mathrm{zAG}}$, we build a new statistically hiding commitment scheme, a conceptually simple cryptographic accumulator, and a computationally efficient chameleon hash function. To gain confidence in Conjecture 10 it would be interesting to prove it in the generic ring model [1]. We leave that for future work.

Acknowledgments. We are grateful to Bjorn Poonen for information about injective polynomials, to Steven Galbraith and Antoine Joux for comments on our cryptographic assumptions, and to Don Zagier for recounting his rationale 
for conjecturing the injectivity of $f(x, y)=x^{7}+3 y^{7}$ over $\mathbb{Q}$. We thank Joe Zimmerman for helpful conversations about this work. This work was supported by DARPA, an NSF research grant, and an NSF Graduate Research Fellowship under Grant No. DGE-114747.

\section{References}

1. Aggarwal, D., Maurer, U.: Breaking RSA generically is equivalent to factoring. In: Joux, A. (ed.) EUROCRYPT 2009. LNCS, vol. 5479, pp. 36-53. Springer, Heidelberg (2009)

2. Ash, A., Gross, R.: Elliptic Tales: Curves, Counting, and Number Theory. Princeton University Press (2012)

3. Ateniese, G., de Medeiros, B.: Identity-based chameleon hash and applications. In: Juels, A. (ed.) FC 2004. LNCS, vol. 3110, pp. 164-180. Springer, Heidelberg (2004)

4. Barić, N., Pfitzmann, B.: Collision-free accumulators and fail-stop signature schemes without trees. In: Fumy, W. (ed.) EUROCRYPT 1997. LNCS, vol. 1233, pp. 480-494. Springer, Heidelberg (1997)

5. Benaloh, J.C., de Mare, M.: One-way accumulators: A decentralized alternative to digital signatures. In: Helleseth, T. (ed.) EUROCRYPT 1993. LNCS, vol. 765, pp. 274-285. Springer, Heidelberg (1994)

6. Boneh, D., Gentry, C., Hamburg, M.: Space-efficient identity based encryption without pairings. In: FOCS, pp. 647-657 (2007)

7. Browkin, J., Brzeziński, J.: Some remarks on the abc-conjecture. Mathematics of Computation 62(206), 931-939 (1994)

8. Camenisch, J., Lysyanskaya, A.: Dynamic accumulators and application to efficient revocation of anonymous credentials. In: Yung, M. (ed.) CRYPTO 2002. LNCS, vol. 2442, pp. 61-76. Springer, Heidelberg (2002)

9. Camenisch, J., Stadler, M.: Efficient group signature schemes for large groups. In: Kaliski Jr., B.S. (ed.) CRYPTO 1997. LNCS, vol. 1294, pp. 410-424. Springer, Heidelberg (1997)

10. Camenisch, J., Stadler, M.: Proof systems for general statements about discrete logarithms. Tech. Rep. 260, Dept. of Computer Science, ETH Zurich (March 1997)

11. Camenisch, J.: Group Signature Schemes and Payment Systems Based on the Discrete Logarithm Problem. Ph.D. thesis, Swiss Federal Institute of Technology Zürich (ETH Zürich) (1998)

12. Catalano, D., Gennaro, R., Howgrave-Graham, N., Nguyen, P.Q.: Paillier's cryptosystem revisited. In: ACM Conference on Computer and Communications Security, pp. 206-214 (2001)

13. Cornelissen, G.: Stockage diophantien et hypothese abc généralisée. Comptes Rendus de l'Académie des Sciences-Series I-Mathematics 328(1), 3-8 (1999)

14. Damgård, I.B.: The Application of Claw Free Functions in Cryptography. Ph.D. thesis, Aarhus University (May 1988)

15. Damgård, I.B.: A design principle for hash functions. In: Brassard, G. (ed.) CRYPTO 1989. LNCS, vol. 435, pp. 416-427. Springer, Heidelberg (1990)

16. Gennaro, R., Halevi, S., Rabin, T.: Secure hash-and-sign signatures without the random oracle. In: Stern, J. (ed.) EUROCRYPT 1999. LNCS, vol. 1592, pp. 123-139. Springer, Heidelberg (1999)

17. Goldwasser, S., Micali, S., Rivest, R.L.: A digital signature scheme secure against adaptive chosen-message attacks. SIAM Journal on Computing 17(2), 281-308 (1988) 
18. Hindry, M., Silverman, J.H.: Diophantine geometry: an introduction, vol. 201. Springer (2000)

19. Kilian, J., Petrank, E.: Identity escrow. In: Krawczyk, H. (ed.) CRYPTO 1998. LNCS, vol. 1462, pp. 169-185. Springer, Heidelberg (1998)

20. Krawczyk, H., Rabin, T.: Chameleon hashing and signatures. In: NDSS, pp. 143-154 (2000)

21. Merkle, R.C.: A digital signature based on a conventional encryption function. In: Pomerance, C. (ed.) CRYPTO 1987. LNCS, vol. 293, pp. 369-378. Springer, Heidelberg (1988)

22. Merkle, R.C.: One way hash functions and DES. In: Brassard, G. (ed.) CRYPTO 1989. LNCS, vol. 435, pp. 428-446. Springer, Heidelberg (1990)

23. Micali, S., Rabin, M., Kilian, J.: Zero-knowledge sets. In: FOCS, pp. 80-91 (2003)

24. Miers, I., Garman, C., Green, M., Rubin, A.D.: Zerocoin: Anonymous distributed e-cash from Bitcoin. IEEE Security and Privacy, 397-411 (2013)

25. Naor, M.: On cryptographic assumptions and challenges. In: Boneh, D. (ed.) CRYPTO 2003. LNCS, vol. 2729, pp. 96-109. Springer, Heidelberg (2003)

26. Ong, H., Schnorr, C.P., Shamir, A.: An efficient signature scheme based on quadratic equations. In: STOC, pp. 208-216 (1984)

27. Pedersen, T.P.: Non-interactive and information-theoretic secure verifiable secret sharing. In: Feigenbaum, J. (ed.) CRYPTO 1991. LNCS, vol. 576, pp. 129-140. Springer, Heidelberg (1992)

28. Pollard, J., Schnorr, C.: An efficient solution of the congruence. IEEE Transactions on Information Theory 33(5), 702-709 (1987)

29. Poonen, B.: Varieties without extra automorphisms III: hypersurfaces. Finite Fields and their Applications 11(2), 230-268 (2005)

30. Poonen, B.: Multivariable polynomial injections on rational numbers. arXiv preprint arXiv:0902.3961v2 (June 2010)

31. Schwenk, J., Eisfeld, J.: Public key encryption and signature schemes based on polynomials over $\mathbb{Z}_{n}$. In: Maurer, U.M. (ed.) EUROCRYPT 1996. LNCS, vol. 1070, pp. 60-71. Springer, Heidelberg (1996)

32. Shallit, J.: An exposition of Pollard's algorithm for quadratic congruences (October 1984)

33. Shamir, A.: On the generation of multivariate polynomials which are hard to factor. In: STOC, pp. 796-804. ACM (1993)

34. Zagier, D.: Personal communication (June 2014)

\section{A Proof of Statistical Hiding}

This appendix presents a proof that the commitment scheme of Section 4.2 is statistically hiding. To demonstrate that the statistical hiding property holds, we show that for any message $m \in \mathbb{Z}_{N}^{*}$, the distribution of the value of a commitment $c$ to $m$ is statistically close to uniform.

The commitment $c$ is generated by sampling a random value $r \leftarrow{ }_{R} \mathbb{Z}_{N}^{*}$ and letting $c \leftarrow m^{7}+3 r^{7}$. Since $r \in \mathbb{Z}_{N}^{*}$, and since $\operatorname{gcd}(7, \phi(N))=1$, the RSA function $f(x)=x^{7} \bmod N$ defines a permutation on $\mathbb{Z}_{N}^{*}$. Thus, there are exactly $\left|\mathbb{Z}_{N}^{*}\right|=\phi(N)$ possible commitments to $m$, and each of these values occurs with equal probability. 
Let the random variable $C$ take on the value of the commitment to $m$ and let $U$ be a random variable uniformly distributed over $\mathbb{Z}_{N}$. Then:

$$
\operatorname{Pr}\left[C=c_{0}\right]=\frac{1}{\phi(N)} ; \quad \operatorname{Pr}\left[U=c_{0}\right]=\frac{1}{N}
$$

The statistical distance between these distributions is:

$$
\begin{aligned}
\Delta(C, U) & =\frac{1}{2} \sum_{c_{0} \in \mathbb{Z}_{N}}\left|\operatorname{Pr}\left[C=c_{0}\right]-\operatorname{Pr}\left[U=c_{0}\right]\right| \\
& =\frac{1}{2} \sum_{c_{0} \in \mathbb{Z}_{N}}\left|\frac{N-\phi(N)}{N \phi(N)}\right|=\frac{(p+q-1)}{2 \phi(N)} \leq \operatorname{negl}(\lambda) .
\end{aligned}
$$

